\title{
Argumentation Based on Classical Logic
}

Philippe Besnard and Anthony Hunter

\section{Introduction}

Argumentation is an important cognitive process for dealing with conflicting information by generating and/or comparing arguments. Often it is based on constructing and comparing deductive arguments. These are arguments that involve some premises (which we refer to as the support of the argument) and a conclusion (which we refer to as the claim of the argument) such that the support deductively entails the claim.

In order to formalize argumentation, we could potentially use any logic to define the logical entailment of the claim from the support. Possible logics include defeasible logics, description logics, paraconsistent logics, modal logics, and classical logic. In this chapter, we focus on deductive arguments in the setting of classical logic. Hence, our starting position is that a deductive argument consists of a claim entailed by a collection of statements such that the claim as well as the statements are denoted by formulae of classical logic and entailment is deduction in classical logic. Classical logic is a well-known formalism. It is widely used in philosophy, mathematics, and computer science for capturing deductive reasoning. It has a simple and intuitive syntax and semantics, and it is supported by a proof theory and extensive foundational results. By using classical logic, we can provide a simple and efficient formalization of argument and counterargument.

So in our framework, an argument is simply a pair $\langle\Phi, \alpha\rangle$ where the first item in the pair is a minimal consistent set of formulae that proves the second item. That is, we account for the support and the claim of an argument though we do not indicate the method of inference since it does not differ from one argument to another: We

Philippe Besnard

IRIT, Universite Paul Sabatier, Toulouse, France

Anthony Hunter

Department of Computer Science, University College London, London, UK 
only consider deductive arguments, hence the method of inference for each and every argument is always entailment according to classical logic.

A counterargument for an argument $\langle\Phi, \alpha\rangle$ is an argument $\langle\Psi, \beta\rangle$ where the claim $\beta$ contradicts the support $\Phi$. Furthermore, we identify a particular kind of counterargument called a canonical undercut $\langle\Psi, \beta\rangle$ where $\beta$ is equivalent to $\neg\left(\phi_{1} \wedge\right.$ ..$\left.\wedge \phi_{n}\right)$ and $\left\{\phi_{1}, \ldots, \phi_{n}\right\}$ is the support of the argument being undercut. This is a valuable form of undercut since it subsumes many other kinds of undercut, and hence focusing on only canonical undercuts renders the presentation and evaluation of counterarguments as a more manageable process.

Each undercut to an argument is itself an argument, and so may be undercut, and hence by recursion each undercut needs to be considered for its undercuts. Exploring systematically the universe of arguments in order to present an exhaustive synthesis of the relevant chains of undercuts for a given argument is the basic principle of our approach.

Following on from the idea that we can capture undercuts, and by recursion undercuts to undercuts, our notion of an argument tree is that it is a synthesis of all the arguments that challenge the argument at the root of the tree, and it also contains all counterarguments that challenge these arguments and so on recursively. In each instance, the only counterarguments we consider are the canonical undercuts.

In the rest of this chapter, we formalize and illustrate arguments and counterarguments (including canonical undercuts), and show how these can be collected into argument trees. We conclude the chapter with a comparison with other approaches to formalising argumentation. Since the aim of this chapter is to just introduce some of the basic ideas to argumentation based on classical logic, the interested reader is requested to refer to $[3,6]$ for more details including formal results.

\section{Preliminaries}

We assume the reader has some knowledge of classical logic. We will represent atoms by lower case roman letters $(a, b, c, d, \ldots)$, formulae by greek letters $(\alpha, \beta, \gamma$, $\ldots .$.$) , and use \wedge, \vee, \rightarrow$, and $\neg$ to denote the logical connectives conjunction, disjunction, negation, and implication (respectively). We use $\vdash$ to denote the classical consequence relation, and so if $\Delta$ is a knowledgebase, and $\alpha$ is a formula, then $\Delta \vdash \alpha$ denotes that $\Delta$ entails $\alpha$ (or equivalently $\alpha$ is a consequence of $\Delta$ ). We also use $\perp$ to denote a contradiction, and so $\Delta \vdash \perp$ denotes that $\Delta$ is contradictory (or equivalently inconsistent).

For the knowledgebase, we first assume a fixed $\Delta$ (a finite set of formulae) and use this $\Delta$ throughout. So when we consider arguments and counterarguments, they will be formed from this $\Delta$. For examples, we will explicitly give the elements of the knowledgebase.

We further assume that every subset of $\Delta$ is given an enumeration $\left\langle\alpha_{1}, \ldots, \alpha_{n}\right\rangle$ of its elements, which we call its canonical enumeration. This really is not a demanding constraint: In particular, the constraint is satisfied whenever we impose an arbitrary 
total ordering over $\Delta$. Importantly, the order has no meaning and is not meant to represent any respective importance of formulae in $\Delta$. It is only a convenient way to indicate the order in which we assume the formulae in any subset of $\Delta$ are conjoined to make a formula logically equivalent to that subset.

The paradigm for our approach is a large repository of information, represented by $\Delta$, from which arguments can be constructed for and against arbitrary claims. Apart from information being understood as declarative statements, there is no $a$ priori restriction on the contents and the pieces of information in the repository can be arbitrarily complex. Therefore, $\Delta$ is not expected to be consistent. It need not even be the case that individual formulae in $\Delta$ are consistent.

The formulae in $\Delta$ can represent certain or uncertain information, and they can represent objective, subjective, or hypothetical statements. So $\Delta$ can represent facts, beliefs, views, ... Furthermore, the items in $\Delta$ can be beliefs from different agents who need not even have the same opinions. It can indeed be the case that an argument formed from such a $\Delta$ takes advantage of partial views from different agents. In any case, it is quite possible for $\Delta$ to have two or more formulae which are logically equivalent ( $\Delta$ can be such that it contains both $\alpha \vee \beta$ and $\beta \vee \alpha$ for example). But wherever they come from, all formulae in $\Delta$ are on a par and treated equitably.

Note, we do not assume any meta-level information about formulae. In particular, we do not assume some preference ordering or "certainty ordering" over formulae. This is in contrast to numerous proposals for argumentation which do assume some form of ordering over formulae. Such orderings can be useful to resolve conflicts by, for example, selecting formulae from a more reliable source. However, this, in a sense, pushes the problem of dealing with conflicting information to one of finding and using orderings over formulae, and as such raises further questions such as: Where does the knowledge about reliability of the sources come from? How can it be assessed? How can it be validated? Besides, reliability is not universal, it usually comes in specialized domains.

This is not to say priorities (or indeed other forms of meta-level information) are not useful. Indeed it is important to use them in some situations when they are available, but we believe that to understand the elements of argumentation, we need to avoid drawing on them - we need to have a comprehensive framework for argumentation that works without recourse to priorities over formulae.

\section{Arguments}

We adopt a very common intuitive notion of an argument and consider some of the ramifications of the definition. Essentially, an argument is a set of appropriate formulae that can be used to classically prove some claim, together with that claim (formulae represent statements, including claims).

Definition 1. An argument is a pair $\langle\Phi, \alpha\rangle$ such that

1. $\Phi \nvdash \perp$. 
2. $\Phi \vdash \alpha$.

3. $\Phi$ is a minimal subset of $\Delta$ satisfying 2 .

If $A=\langle\Phi, \alpha\rangle$ is an argument, we say that $A$ is an argument for $\alpha$ (which in general is not an element of $\Delta$ ) and we also say that $\Phi$ is a support for $\alpha$. We call $\alpha$ the claim of the argument and we call $\Phi$ the support of the argument.

Example 1. Let $\Delta=\{a, a \rightarrow b, c \rightarrow \neg b, c, d, d \rightarrow b, \neg a, \neg c\}$. Some arguments are:

$$
\begin{gathered}
\langle\{a, a \rightarrow b\}, b\rangle \\
\langle\{c \rightarrow \neg b, c\}, \neg b\rangle \\
\langle\{d, d \rightarrow b\}, b\rangle \\
\langle\{\neg a\}, \neg a\rangle \\
\langle\{\neg c\}, \neg c\rangle \\
\langle\{a \rightarrow b\}, \neg a \vee b\rangle \\
\langle\{\neg c\}, d \rightarrow \neg c\rangle
\end{gathered}
$$

The need for the first condition of Definition 1 can be illustrated by means of the next example.

Example 2. Consider the following atoms.

$a$ The office phone number is 02045458721

$b$ I am a billionaire

Now let $\{a, \neg a\} \subseteq \Delta$, and so by classical logic, we have

$$
\{a, \neg a\} \vdash b
$$

However, we do not want to have $\{a, \neg a\}$ as the support for an argument with claim $b$. If we were to allow that as an argument, then we would have an argument with this support and with any claim in the language. Hence, if we were to allow inconsistent supports, then we would have an overwhelming number of useless arguments.

The second condition of Definition 1 aims at ensuring that the support is sufficient for the consequent to hold, as is illustrated in the next example.

Example 3. Consider the informal argument which is acceptable.

It is an even number, and therefore we can infer it is not an odd number.

Now consider the following atoms.

$e$ It is an even number

$o$ It is an odd number

So we can represent the premise of informal argument by the set $\{e\}$. However, by classical logic we have that $\{e\} \forall \neg o$, and hence the following is not an argument. 


$$
\langle\{e\}, \neg o\rangle
$$

If we want to turn the informal argument (which is an enthymeme) into an argument, we need to make explicit all the premises. So we can represent the above informal argument by the following formal argument.

$$
\langle\{e, \neg e \vee \neg o\}, \neg o\rangle
$$

An enthymeme is a form of reasoning in which some premises are implicit, most often because they are obvious. As another example, "The baby no longer has her parents, therefore she is an orphan" (in symbols, $\neg p$ hence $o$ ) is an enthymeme: The reasoning is correct despite omitting the trivial premise stating that "if a baby no longer has her parents, then she is an orphan" (in symbols, $\{\neg p, \neg p \rightarrow o\} \vdash o$ ).

Minimality (i.e., condition 3 Definition 1) is not an absolute requirement, although some properties depend on it. Importantly, the condition is not of a mere technical nature.

Example 4. Consider the following formulae.

$p \quad$ I like paprika

$r \quad$ It is raining

$r \rightarrow q$ If it is raining, then I should use my umbrella

It is possible to argue that "I should use my umbrella, because I should use my umbrella, if it is raining, and indeed it is", to be captured formally by the argument

$$
\langle\{r, r \rightarrow q\}, q\rangle
$$

In contrast, it is counter-intuitive to argue that "I should use my umbrella, because I like paprika and I should use my umbrella, if it is raining, and indeed it is", to be captured formally by

$$
\langle\{p, r, r \rightarrow q\}, q\rangle
$$

which fails to be an argument because condition 3 is not satisfied.

The underlying idea for condition 3 is that an argument makes explicit the connection between reasons for a claim and the claim itself. But that would not be the case if the reasons were not exactly identified. In other words, if reasons incorporated irrelevant information and so included formulae not used in the proof of the claim.

Arguments are not necessarily independent. In a sense, some encompass others (possibly up to some form of equivalence), which is the topic we now turn to.

Definition 2. An argument $\langle\Phi, \alpha\rangle$ is more conservative than an argument $\langle\Psi, \beta\rangle$ iff $\Phi \subseteq \Psi$ and $\beta \vdash \alpha$.

Example 5. $\langle\{a\}, a \vee b\rangle$ is more conservative than $\langle\{a, a \rightarrow b\}, b\rangle$.

Roughly speaking, a more conservative argument is more general: It is, so to speak, less demanding on the support and less specific about the consequent. 
Example 6. Consider the following atoms.

$p$ The number is divisible by 10

$q$ The number is divisible by 2

$r$ The number is an even number

We use these for the following set of formulae.

$$
\Delta=\{p, p \rightarrow q, q \rightarrow r\}
$$

Hence, the following is an argument with the claim "The number is divisible by 2".

$$
\langle\{p, p \rightarrow q\}, q\rangle
$$

Similarly, the following is argument with claim "The number is divisible by 2 and the number is an even number".

$$
\langle\{p, p \rightarrow q, q \rightarrow r\}, r \wedge q\rangle
$$

However, the first argument $\langle\{p, p \rightarrow q\}, q\rangle$ is more conservative than the second argument $\langle\{p, p \rightarrow q, q \rightarrow r\}, r \wedge q\rangle$ which can be retrieved from it:

$$
\left.\begin{array}{l}
\langle\{p, p \rightarrow q\}, q\rangle \\
\{q, q \rightarrow r\} \mid=r \wedge q
\end{array}\right\} \Rightarrow\langle\{p, p \rightarrow q, q \rightarrow r\}, r \wedge q\rangle
$$

We will use the notion of "more conservative" to help us identify the most useful counterarguments amongst the potentially large number of counterarguments.

\section{Counterarguments}

Informally, an argument that disagrees with another argument is described as a counterargument. So counterarguments are an important part of the argumentation process. They highlight points of contention.

In logic-based approaches to argumentation, an intuitive notion of counterargument is captured with the idea of defeaters, which are arguments whose claim refutes the support of another argument [23, 14, 19, 25, 24, 22]. This gives us a general way for an argument to challenge another.

Definition 3. A defeater for an argument $\langle\Phi, \alpha\rangle$ is an argument $\langle\Psi, \beta\rangle$ such that $\beta \vdash \neg\left(\phi_{1} \wedge \ldots \wedge \phi_{n}\right)$ for some $\left\{\phi_{1}, \ldots, \phi_{n}\right\} \subseteq \Phi$.

Example 7. Let $\Delta=\{\neg a, a \vee b, a \leftrightarrow b, c \rightarrow a\}$. Then, $\langle\{a \vee b, a \leftrightarrow b\}, a \wedge b\rangle$ is a defeater for $\langle\{\neg a, c \rightarrow a\}, \neg c\rangle$. A more conservative defeater for $\langle\{\neg a, c \rightarrow a\}, \neg c\rangle$ is $\langle\{a \vee b, a \leftrightarrow b\}, a \vee c\rangle$. 
The notion of assumption attack to be found in the literature is less general than the above notion of defeater, of which special cases are undercut and rebuttal as discussed next.

Some arguments directly oppose the support of others, which amounts to the notion of an undercut.

Definition 4. An undercut for an argument $\langle\Phi, \alpha\rangle$ is an argument $\left\langle\Psi, \neg\left(\phi_{1} \wedge \ldots \wedge\right.\right.$ $\left.\left.\phi_{n}\right)\right\rangle$ where $\left\{\phi_{1}, \ldots, \phi_{n}\right\} \subseteq \Phi$.

Example 8. Let $\Delta=\{a, a \rightarrow b, c, c \rightarrow \neg a\}$. Then, $\langle\{c, c \rightarrow \neg a\}, \neg(a \wedge(a \rightarrow b))\rangle$ is an undercut for $\langle\{a, a \rightarrow b\}, b\rangle$. A less conservative undercut for $\langle\{a, a \rightarrow b\}, b\rangle$ is $\langle\{c, c \rightarrow \neg a\}, \neg a\rangle$.

The most direct form of a conflict between arguments is when two arguments have opposite claims. This case is captured in the literature through the notion of a rebuttal.

Definition 5. An argument $\langle\Psi, \beta\rangle$ is a rebuttal for an argument $\langle\Phi, \alpha\rangle$ iff $\beta \leftrightarrow \neg \alpha$ is a tautology.

Example 9. Consider a discussion in a newspaper editorial office about whether or not to proceed with the publication of some indiscretion about a prominent politician. Suppose the key bits of information are captured by the following five statements.

$p \quad$ Simon Jones is a Member of Parliament

$p \rightarrow \neg q$ If Simon Jones is a Member of Parliament then we need not keep quiet about details of his private life

$r \quad$ Simon Jones just resigned from the House of Commons

$r \rightarrow \neg p$ If Simon Jones just resigned from the House of Commons then he is not a Member of Parliament

$\neg p \rightarrow q$ If Simon Jones is not a Member of Parliament then we need to keep quiet about details of his private life

The first two statements form an argument $A$ whose claim is that we can publicize details about his private life. The next two statements form an argument whose claim is that he is not a Member of Parliament (contradicting an item in the support of $A$ ) and that is a counterargument against $A$. The last three statements combine to give an argument whose claim is that we cannot publicize details about his private life (contradicting the claim of $A$ ) and that, too, is a counterargument against $A$. In symbols, we obtain the following argument (below left), and counterarguments (below right).

$$
\langle\{p, p \rightarrow \neg q\}, \neg q\rangle \quad\left\{\begin{array}{l}
\text { An undercut is }\langle\{r, r \rightarrow \neg p\}, \neg p\rangle \\
\text { A rebuttal is }\langle\{r, r \rightarrow \neg p, \neg p \rightarrow q\}, q\rangle
\end{array}\right.
$$

Trivially, undercuts are defeaters but it is also quite simple to establish that rebuttals are defeaters. Furthermore, if an argument has defeaters then it has undercuts, 
naturally. It may happen that an argument has defeaters but no rebuttals as illustrated next.

Example 10. Let $\Delta=\{a \wedge b, \neg b\}$. Then, $\langle\{a \wedge b\}, a\rangle$ has at least one defeater but no rebuttal.

There are some important differences between rebuttals and undercuts that can be seen in the following examples. In the first, we see how an undercut for an argument need not be a rebuttal for that argument, and in the second, we see how rebuttal for an argument need not be an undercut for that argument.

Example 11. $\langle\{\neg a\}, \neg a\rangle$ is an undercut for $\langle\{a, a \rightarrow b\}, b\rangle$ but is not a rebuttal for it. Clearly, $\langle\{\neg a\}, \neg a\rangle$ does not rule out $b$. Actually, an undercut may even agree with the claim of the objected argument: $\langle\{b \wedge \neg a\}, \neg a\rangle$ is an undercut for $\langle\{a, a \rightarrow$ $b\}, b\rangle$. In this case, we have an argument with an undercut that conflicts with the support of the argument but implicitly provides an alternative way to deduce the claim of the argument. This should make it clear that an undercut need not question the claim of an argument but only the reason(s) given by that argument to support its claim. Of course, there are also undercuts that challenge an argument on both counts: Just consider $\langle\{\neg a \wedge \neg b\}, \neg a\rangle$ which is such an undercut for the argument $\langle\{a, a \rightarrow b\}, b\rangle$.

Example 12. $\langle\{\neg b\}, \neg b\rangle$ is a rebuttal for $\langle\{a, a \rightarrow b\}, b\rangle$ but is not an undercut for it because $b$ is not in $\{a, a \rightarrow b\}$. Observe that there is not even an argument equivalent to $\langle\{\neg b\}, \neg b\rangle$ which would be an undercut for $\langle\{a, a \rightarrow b\}, b\rangle$ : In order to be an undercut for $\langle\{a, a \rightarrow b\}, b\rangle$, an argument should be of the form $\langle\Phi, \neg a\rangle,\langle\Phi, \neg(a \rightarrow$ $b)\rangle$ or $\langle\Phi, \neg(a \wedge(a \rightarrow b))\rangle$ but $\neg b$ is not logically equivalent to $\neg a, \neg(a \rightarrow b)$ or $\neg(a \wedge(a \rightarrow b))$.

Both undercuts and rebuttals are useful kinds of counterargument. However, we will see in the next section that we can effectively capture all we need to know about the counterarguments to an argument by just using a special kind of undercut called a canonical undercut.

\section{Canonical undercuts}

A particularly useful kind of undercut is the maximally conservative undercut which we define next.

Definition 6. $\langle\Psi, \beta\rangle$ is a maximally conservative undercut of $\langle\Phi, \alpha\rangle$ iff for all undercuts $\left\langle\Psi^{\prime}, \beta^{\prime}\right\rangle$ of $\langle\Phi, \alpha\rangle$, if $\Psi^{\prime} \subseteq \Psi$ and $\beta \vdash \beta^{\prime}$ then $\Psi \subseteq \Psi^{\prime}$ and $\beta^{\prime} \vdash \beta$.

Evidently, $\langle\Psi, \beta\rangle$ is a maximally conservative undercut of $\langle\Phi, \alpha\rangle$ iff $\langle\Psi, \beta\rangle$ is an undercut of $\langle\Phi, \alpha\rangle$ such that no undercuts of $\langle\Phi, \alpha\rangle$ are strictly more conservative than $\langle\Psi, \beta\rangle$. 
The next example shows that a collection of counterarguments to the same argument can sometimes be summarized in the form of a single maximally conservative undercut of the argument, thereby avoiding some amount of redundancy among counterarguments.

Example 13. Consider the following formulae concerning who is going to a party.

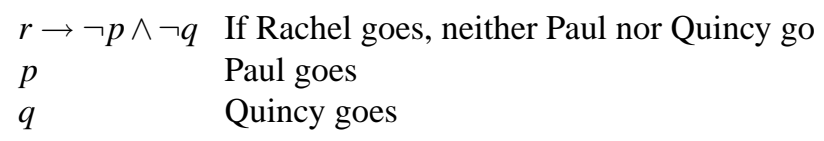

Hence both Paul and Quincy go (initial argument)

$$
\langle\{p, q\}, p \wedge q\rangle
$$

Now assume the following additional piece of information

$$
r \quad \text { Rachel goes }
$$

Hence Paul does not go (a first counterargument)

$$
\langle\{r, r \rightarrow \neg p \wedge \neg q\}, \neg p\rangle
$$

Hence Quincy does not go (a second counterargument)

$$
\langle r, r \rightarrow \neg p \wedge \neg q\}, \neg q\rangle
$$

A maximally conservative undercut (for the initial argument) that subsumes both counterarguments above is

$$
\langle\{r, r \rightarrow \neg p \wedge \neg q\}, \neg(p \wedge q)\rangle
$$

The fact that the maximally conservative undercut in Example 13 happens to be a rebuttal of the argument is only accidental. Actually, the claim of a maximally conservative undercut for an argument is exactly the negation of the full support of the argument. In other words, if $\left\langle\Psi, \neg\left(\phi_{1} \wedge \ldots \wedge \phi_{n}\right)\right\rangle$ is a maximally conservative undercut for an argument $\langle\Phi, \alpha\rangle$, then $\Phi=\left\{\phi_{1}, \ldots, \phi_{n}\right\}$.

Note that if $\left\langle\Psi, \neg\left(\phi_{1} \wedge \ldots \wedge \phi_{n}\right)\right\rangle$ is a maximally conservative undercut for an argument $\langle\Phi, \alpha\rangle$, then so are $\left\langle\Psi, \neg\left(\phi_{2} \wedge \ldots \wedge \phi_{n} \wedge \phi_{1}\right)\right\rangle$ and $\left\langle\Psi, \neg\left(\phi_{3} \wedge \ldots \wedge \phi_{n} \wedge \phi_{1} \wedge\right.\right.$ $\left.\left.\phi_{2}\right)\right\rangle$ and so on. However, they are all identical (in the sense that each is more conservative than the others). We can ignore the unnecessary variants by just considering the canonical undercuts defined as follows.

Definition 7. An argument $\left\langle\Psi, \neg\left(\phi_{1} \wedge \ldots \wedge \phi_{n}\right)\right\rangle$ is a canonical undercut for $\langle\Phi, \alpha\rangle$ iff it is an undercut for $\langle\Phi, \alpha\rangle$ and $\left\langle\phi_{1}, \ldots, \phi_{n}\right\rangle$ is the canonical enumeration of $\Phi$.

Recall (from the Preliminaries section) that the ordering given by the canonical enumeration has no meaning and is not meant to represent any respective importance of formulae in $\Delta$. It is only a convenient way to indicate the order in which we assume the formulae in any subset of $\Delta$ are conjoined to make a formula logically equivalent to that subset. 
Example 14. Returning to Example 13, suppose the canonical enumeration is as follows.

$$
\langle r, p, r \rightarrow \neg p \wedge \neg q, q\rangle
$$

Then both the following are maximally conservative undercuts, but only the first is a canonical undercut.

$$
\begin{aligned}
& \langle\{r, r \rightarrow \neg p \wedge \neg q\}, \neg(p \wedge q)\rangle \\
& \langle\{r, r \rightarrow \neg p \wedge \neg q\}, \neg(q \wedge p)\rangle
\end{aligned}
$$

The nice feature of canonical undercuts is that they are all maximally conservative undercuts. In other words, an argument $\left\langle\Psi, \neg\left(\phi_{1} \wedge \ldots \wedge \phi_{n}\right)\right\rangle$ is a canonical undercut for $\langle\Phi, \alpha\rangle$ iff it is a maximally conservative undercut for $\langle\Phi, \alpha\rangle$ and $\left\langle\phi_{1}, \ldots, \phi_{n}\right\rangle$ is the canonical enumeration of $\Phi$.

Clearly, an argument may have more than one canonical undercut. This raises the question of how do the canonical undercuts for the same argument look like, and how do they differ from one another? In response to the first question, any two different canonical undercuts for the same argument have the same claim, but distinct supports, and in response to the second question, given two different canonical undercuts for the same argument, none is more conservative than the other.

Example 15. Let $\Delta=\{a, b, \neg a, \neg b\}$. Both the following are canonical undercuts for $\langle\{a, b\}, a \leftrightarrow b\rangle$, but neither is more conservative than the other.

$$
\begin{aligned}
& \langle\{\neg a\}, \neg(a \wedge b)\rangle \\
& \langle\{\neg b\}, \neg(a \wedge b)\rangle
\end{aligned}
$$

A further important property of canonical undercuts is the following which shows how they give us the useful information concerning counterarguments for an argument: For each defeater $\langle\Psi, \beta\rangle$ of an argument $\langle\Phi, \alpha\rangle$, there exists a canonical undercut for $\langle\Phi, \alpha\rangle$ that is more conservative than $\langle\Psi, \beta\rangle$. Therefore, the set of all canonical undercuts of an argument represent all the defeaters of that argument (informally, all its counterarguments). This is to be taken advantage of in the next section.

\section{Argument trees}

How does argumentation usually take place? Argumentation starts when an initial argument is put forward, making some claim. An objection is raised, in the form of a counterargument. The latter is addressed in turn, eventually giving rise to a counter-counterargument, if any. And so on. However, there often is more than one counterargument to the initial argument, and if the counterargument actually raised in the first place had been different, the counter-counterargument would have been different, too, and similarly the counter-counter-counterargument, if any, and so on, and hence the argumentation would have taken a possibly quite different course. 
So do we find all the alternative courses which could take place from a given initial argument? And is it possible to represent them in a rational way? Let alone the most basic question: How do we make sure that no further counterargument can be expressed from the information available?

Example 16. Let the following be our knowledgebase.

$$
\{a, b, c, \neg a \vee \neg b \vee \neg c\}
$$

Suppose we start with the following argument.

$$
\langle\{a, b, c\}, a \wedge b \wedge c\rangle
$$

Now we have numerous undercuts to this argument including the following.

$$
\begin{aligned}
& \langle\{b, c, \neg a \vee \neg b \vee \neg c\}, \neg a\rangle \\
& \langle\{a, c, \neg a \vee \neg b \vee \neg c\}, \neg b\rangle \\
& \langle\{a, b, \neg a \vee \neg b \vee \neg c\}, \neg c\rangle \\
& \langle\{a, \neg a \vee \neg b \vee \neg c\}, \neg b \vee \neg c\rangle \\
& \langle\{b, \neg a \vee \neg b \vee \neg c\}, \neg a \vee \neg c\rangle \\
& \langle\{c, \neg a \vee \neg b \vee \neg c\}, \neg a \vee \neg b\rangle \\
& \langle\{\neg a \vee \neg b \vee \neg c\}, \neg a \vee \neg b \vee \neg c\rangle
\end{aligned}
$$

All these undercuts say the same thing which is that the set $\{a, b, c\}$ is inconsistent together with the formula $\neg a \vee \neg b \vee \neg c$. As a result, this can be captured by the last undercut listed above. Note this is the maximally conservative undercut amongst the undercuts listed, and moreover it is a canonical undercut. This example therefore illustrates how the canonical undercuts are the undercuts that represent all the other undercuts.

Often each undercut may itself be undercut, as illustrated by the next example.

Example 17. Consider the following atoms that concern the debate on addressing the shortage of oil with biofuel and thereby addressing the effect of the shortage on inflation. However, when grain is used to produce biofuel, then this can cause inflation because it causes shortages of grain for food. But, biofuel can be produced from the large unexploited sources of biowaste (such as the remainder of the wheat plant after the grain has been removed).

$o$ there is decreased availability of oil

$b$ there is increased production of biofuel

$g$ there is decreased availability of food grain

$i$ there is increased inflation of prices

$w$ there is unexploited availability of biowaste

We can use these for the following formulae.

$$
\{o, o \rightarrow b, b \rightarrow g, b \rightarrow \neg i, g \rightarrow i, w, w \rightarrow b \wedge \neg g\}
$$


Now we can form the the following argument to capture the first part of the above discussion.

$$
\langle\{o, o \rightarrow b, b \rightarrow \neg i\}, \neg i\rangle
$$

The following canonical undercut captures the counterargument to the above.

$$
\langle\{o, o \rightarrow b, b \rightarrow g, g \rightarrow i\}, \neg(o \wedge(o \rightarrow b) \wedge(b \rightarrow \neg i))\rangle
$$

We can capture the counterargument to the above undercut by the following canonical undercut.

$$
\langle\{w, w \rightarrow b \wedge \neg g\}, \neg(o \wedge(o \rightarrow b) \wedge(b \rightarrow g) \wedge(g \rightarrow i))\rangle
$$

So we can represent the above discussion by three arguments, where we start with an argument, we have an undercut to that argument, and an undercut to the undercut.

It is also common to have more than one canonical undercut for an argument. Multiple undercuts are illustrated by the following example.

Example 18. Suppose we have the following knowledgebase.

$$
\{(\neg r \rightarrow \neg p) \rightarrow \neg q,(p \rightarrow s) \rightarrow \neg \neg q, r, s, \neg p\}
$$

Also suppose we construct the following argument.

$$
\langle\{r,(\neg r \rightarrow \neg p) \rightarrow \neg q\}, \neg q\rangle
$$

Then the following two arguments are each a canonical undercut to the above argument.

$$
\begin{gathered}
\langle\{s,(p \rightarrow s) \rightarrow \neg \neg q\}, \neg(r \wedge(\neg r \rightarrow \neg p) \rightarrow \neg q)\rangle \\
\langle\{\neg p,(p \rightarrow s) \rightarrow \neg \neg q\}, \neg(r \wedge(\neg r \rightarrow \neg p) \rightarrow \neg q)\rangle
\end{gathered}
$$

Finally, the following argument is a canonical undercut to the first of the above canonical undercuts.

$$
\langle\{\neg p,(\neg r \rightarrow \neg p) \rightarrow \neg q\}, \neg(s \wedge(p \rightarrow s) \rightarrow \neg \neg q)\rangle
$$

We now turn to one final issue before we formalize the notion of argument trees.

Example 19. Argumentation sometimes falls into a repetitive and uninformative cycle as illustrated below with a case of the "Chicken and Egg dilemma".

$$
\begin{aligned}
& \text { Dairyman: }- \text { Egg was first } \\
& \text { Farmer: } \quad \text { - Chicken was first } \\
& \text { Dairyman: }- \text { Egg was first } \\
& \text { Farmer: } \quad \text { - Chicken was first } \\
& \begin{array}{cl}
\ldots & -\ldots
\end{array}
\end{aligned}
$$

The following propositional atoms are introduced: 
$p$ Egg was first

$q$ Chicken was first

$r$ The chicken comes from the egg

$s$ The egg comes from the chicken

It can be assumed that the chicken was first and that the egg was first are not equivalent. (i.e. $\neg(p \leftrightarrow q)$ ). Also, it can be assumed that the egg comes from the chicken (i.e. $s$ ) and the chicken comes from the egg (i.e. $r$ ). Moreover, if the egg comes from the chicken then the egg was not first. (i.e. $s \rightarrow \neg p$ ). Similarly, if the chicken comes from the egg then the chicken was not first (i.e. $r \rightarrow \neg q$ ). Then, the above dispute can be represented as follows:

$$
\begin{aligned}
& \langle\{s \rightarrow \neg p, s, \neg(p \leftrightarrow q)\}, q\rangle \\
& \langle\{r \rightarrow \neg q, r, \neg(p \leftrightarrow q)\}, p\rangle \\
& \uparrow \\
& \langle\{s \rightarrow \neg p, s, \neg(p \leftrightarrow q)\}, q\rangle \\
& \uparrow \\
& \langle\{r \rightarrow \neg q, r, \neg(p \leftrightarrow q)\}, p\rangle \\
& \uparrow
\end{aligned}
$$

We are now ready for our definition (below) of an argument tree in which the root of the tree is an argument of interest, and the children for any node are the canonical undercuts for that node. In the definition, we avoid the circularity seen in the above example by incorporating an intuitive constraint.

Definition 8. An argument tree for $\alpha$ is a tree where the nodes are arguments such that

1. The root is an argument for $\alpha$.

2. For no node $\langle\Phi, \beta\rangle$ with ancestor nodes $\left\langle\Phi_{1}, \beta_{1}\right\rangle, \ldots,\left\langle\Phi_{n}, \beta_{n}\right\rangle$ is $\Phi$ a subset of $\Phi_{1} \cup \cdots \cup \Phi_{n}$.

3. The children nodes of a node $N$ consist of all canonical undercuts for $N$ that obey 2.

We illustrate the definition of an argument tree in the following examples.

Example 20. Returning to Example 9, we have the following five formulae.

$p \quad$ Simon Jones is a Member of Parliament

$p \rightarrow \neg q$ If Simon Jones is a Member of Parliament then we need not keep quiet about details of his private life

$r \quad$ Simon Jones just resigned from the House of Commons

$r \rightarrow \neg p$ If Simon Jones just resigned from the House of Commons then he is not a Member of Parliament

$\neg p \rightarrow q$ If Simon Jones is not a Member of Parliament then we need to keep quiet about details of his private life 
These can be used to construct the argument tree below.

$$
\begin{gathered}
\langle\{p, p \rightarrow \neg q\}, \neg q\rangle \\
\uparrow \\
\langle\{r, r \rightarrow \neg p\}, \neg(p \wedge(p \rightarrow \neg q))\rangle
\end{gathered}
$$

Example 21. Given $\Delta=\{a, a \rightarrow b, c, c \rightarrow \neg a, \neg c \vee \neg a\}$, we have the following argument tree.

$$
\begin{gathered}
\langle\{a, a \rightarrow b\}, b\rangle \\
\langle\{c, c \rightarrow \neg a\}, \neg(a \wedge(a \rightarrow b))\rangle \quad \nwarrow \\
\langle\{c, \neg c \vee \neg a\}, \neg(a \wedge(a \rightarrow b))\rangle
\end{gathered}
$$

Note the two undercuts are equivalent. They do count as two arguments because they are based on two different items of the knowledgebase (even though these items turn out to be logically equivalent).

For the rest of this chapter, we adopt a lighter notation, writing $\langle\Psi, \diamond\rangle$ for a canonical undercut of $\langle\Phi, \beta\rangle$. Clearly, $\diamond$ is $\neg\left(\phi_{1} \wedge \ldots \wedge \phi_{n}\right)$ where $\left\langle\phi_{1}, \ldots, \phi_{n}\right\rangle$ is the canonical enumeration for $\Phi$.

Example 22. Consider the following atoms concerning the safety of mobiles phones for children.

$q_{1}$ Mobile phones are safe for children

$q_{2}$ Mobile phones have a health risk

$q_{3}$ Mobile phones heat the brain

$q_{4}$ Mobile phones emit strong electromagnetic radiation

$q_{5}$ There is a high density of phone masts

$q_{6}$ Mobile phones can be used hands-free

$q_{7}$ Hot baths heat the brain

Now suppose we have the following knowledgebase

$$
\left\{\neg q_{2}, \neg q_{2} \rightarrow q_{1}, q_{4}, q_{4} \rightarrow q_{3}, q_{3} \rightarrow q_{2}, q_{5}, q_{5} \rightarrow \neg q_{4}, q_{6}, q_{6} \rightarrow \neg q_{3}, q_{7}, q_{7} \rightarrow \neg q_{2}\right\}
$$

From this knowledgebase, we can obtain the following argument tree.

$$
\begin{gathered}
\left\langle\left\{\neg q_{2}, \neg q_{2} \rightarrow q_{1}\right\}, q_{1}\right\rangle \\
\uparrow \\
\left\langle\left\{\begin{array}{c}
\left\langle\left\{q_{4}, q_{4} \rightarrow q_{3}, q_{3} \rightarrow q_{2}\right\}, \diamond\right\rangle \\
\uparrow
\end{array}\right.\right. \\
\left\langle\left\{q_{5}, q_{5} \rightarrow \neg q_{4}\right\}, \diamond\right\rangle \quad\left\langle\left\{q_{6}, q_{6} \rightarrow \neg q_{3}\right\}, \diamond\right\rangle \quad\left\langle\left\{q_{7}, q_{7} \rightarrow \neg q_{2}\right\}, \diamond\right\rangle
\end{gathered}
$$

We motivate the conditions of Definition 8 as follows: Condition 2 is meant to avoid the situation illustrated by Example 23; and Condition 3 is meant to avoid the situation illustrated by Example 24. 
Example 23. Let $\Delta=\{a, a \rightarrow b, c \rightarrow \neg a, c\}$.

$$
\begin{gathered}
\langle\{a, a \underset{\uparrow}{\rightarrow} b\}, b\rangle \\
\langle\{c, c \underset{\uparrow}{\rightarrow} \neg a\}, \diamond\rangle \\
\left\langle\left\{\begin{array}{c}
\uparrow \\
\langle\{a, c \rightarrow \neg a\}, \diamond\rangle
\end{array}\right.\right.
\end{gathered}
$$

This is not an argument tree because Condition 2 is not met. The undercut to the undercut is actually making exactly the same point (that $a$ and $c$ are incompatible) as the undercut itself does, just by using modus tollens instead of modus ponens.

Example 24. Given $\Delta=\{a, b, a \rightarrow c, b \rightarrow d, \neg a \vee \neg b\}$, consider the following tree.

$$
\langle\{a, \neg a \vee \neg b\}, \neg b\rangle \nearrow^{\langle\{a, b, a \rightarrow c, b \rightarrow d\}, c \wedge d\rangle} \nwarrow\langle\langle\{b, \neg a \vee \neg b\}, \neg a\rangle
$$

This is not an argument tree because the two children nodes are not maximally conservative undercuts. The first undercut is essentially the same argument as the second undercut in a rearranged form (relying on $a$ and $b$ being incompatible, assume one and then conclude that the other doesn't hold). If we replace these by the maximally conservative undercut $\langle\{\neg a \vee \neg b\}, \diamond\rangle$, we obtain an argument tree.

The form of an argument tree is not arbitrary. It summarizes all possible courses of argumentation about the argument in the root node. Each node except the root node is the starting point of an implicit series of related arguments. What happens is that for each possible course of argumentation (from the root), an initial sequence is provided as a branch of the tree up to the point that no subsequent counter ${ }^{n}$-argument needs a new item in its support (where new means not occurring somewhere in that initial sequence). Also, the counterarguments in a course of argumentation may somewhat differ from the ones in the corresponding branch of the argument tree:

Example 25. We return to Example 18 which has the following knowledgebase.

$$
\{(\neg r \rightarrow \neg p) \rightarrow \neg q,(p \rightarrow s) \rightarrow \neg \neg q, r, s, \neg p\}
$$

The argument tree with $\langle\{r,(\neg r \rightarrow \neg p) \rightarrow \neg q\}, \neg q\rangle$ as its root is

$$
\begin{gathered}
\langle\{r,(\neg r \rightarrow \neg p) \rightarrow \neg q\}, \neg q\rangle \\
\nearrow \\
\langle\{s,(p \rightarrow s) \rightarrow \neg \neg q\}, \diamond\rangle \quad\langle\{\neg p,(p \rightarrow s) \rightarrow \neg \neg q\}, \diamond\rangle \\
\langle\{\neg p,(\neg r \rightarrow \neg p) \rightarrow \neg q\}, \diamond\rangle
\end{gathered}
$$

Example 26. We return to the "Chicken and Egg dilemma" presented in Example 19 
Dairyman: - Egg was first

Farmer: - Chicken was first

Dairyman: - Egg was first

Farmer: $\quad-$ Chicken was first

$$
\text { ... }-\ldots
$$

Here are the formulae again:

$p$ Egg was first

$q$ Chicken was first

$r$ The chicken comes from the egg

$s$ The egg comes from the chicken

$\neg(p \leftrightarrow q)$ That the egg was first and that the chicken was first are not equivalent

$r \rightarrow \neg q$ The chicken comes from the egg implies that the chicken was not first

$s \rightarrow \neg p$ The egg comes from the chicken implies that the egg was not first

So, $\Delta=\{\neg(p \leftrightarrow q), r \rightarrow \neg q, s \rightarrow \neg p, r, s\}$. The argument tree with the dairyman's argument as its root is

$$
\begin{gathered}
\langle\{r \rightarrow \neg q, r, \neg(q \leftrightarrow p)\}, p\rangle \\
\uparrow \\
\langle\{s \rightarrow \neg p, s, \neg(q \leftrightarrow p)\}, \diamond\rangle
\end{gathered}
$$

but it does not mean that the farmer has the last word nor that the farmer wins the dispute! The argument tree is merely a representation of the argumentation (in which the dairyman provides the initial argument). Although the argument tree is finite, the argumentation here is infinite and unresolved.

We now consider a widely used criterion in argumentation theory for determining whether the argument at the root of the argument tree is warranted (e.g. [21, 15]). For this, each node is marked as either $U$ for undefeated or $D$ for defeated.

Definition 9. The judge function, denoted Judge, assigns either Warranted or Unwarranted to each argument tree $T$ such that Judge $(T)=$ Warranted iff Mark $\left(A_{r}\right)$ $=U$ where $A_{r}$ is the root node of $T$. For all nodes $A_{i}$ in $T$, if there is child $A_{j}$ of $A_{i}$ such that $\operatorname{Mark}\left(A_{j}\right)=U$, then $\operatorname{Mark}\left(A_{i}\right)=D$, otherwise $\operatorname{Mark}\left(A_{i}\right)=U$.

As a direct consequence of the above definition, the root is undefeated iff all its children are defeated.

Example 27. Returning to Example 22, we see that the root of the tree $T$ is undefeated, and hence Judge $(T)=$ Warranted.

Example 28. Returning to Example 26, we see that the root of the tree $T$ is defeated, and hence Judge $(T)=$ Unwarranted.

In general, a complete argument tree (i.e. an argument tree with all the canonical undercuts for each node as children of that node) provides an efficient representation 
of the arguments and counterarguments. Furthermore, if $\Delta$ is finite, there is a finite number of argument trees with the root being an argument with claim $\alpha$ that can be formed from $\Delta$, and each of these trees has finite branching and a finite depth.

\section{Discussion}

In this chapter, we have reviewed a framework for argumentation based on classical logic. The key features of this framework are the clarification of the nature of arguments and counterarguments, the identification of canonical undercuts which we argue are the only undercuts that we need to take into account, and the representation of argument trees which provide a way of exhaustively collating arguments and counterarguments. This chapter is based on a particular proposal for logic-based argumentation for the propositional case [3, 6]. In comparison with the previous proposals based on classical logic (e.g. [1, 20]), our proposal provides a much more detailed analysis of counterarguments, and ours is the first proposal to consider canonical undercuts. We believe that without the notion of canonical undercuts, the number of undercuts available is unmanageable, and so restricting to canonical undercuts reduces the number of undercuts to consider, and renders it manageable.

It is interesting to compare our approach with other logic-based approaches to argumentation that use logics other than classical logic for the definition of deduction. The most common alternative is a form of defeasible logic such as defeasible logic programming [15], defeasible argumentation with specificity-based preferences [23], and argument-based extended logic programming [21]. For a general coverage of defeasible logics in argumentation see [10, 22, 6].

Whilst there are various positive features of these proposals based on defeasible logic, including computational viability, and modeling of intuitive features of defeasible reasoning, there are complications with these proposals with respect to the interplay between strict rules and defeasible rules, and the interplay between these rules and the use of priorities over rules. This can render the deductive process to be less than transparent. In comparison, we believe that using classical logic provides a simpler and clear notion of deduction, of argument, and of counterargument.

Furthermore, various questions of rationality have been raised concerning the use of defeasible logic for deduction in argumentation [8]. One of the proposals made for rationality is that contrapositive reasoning needs to be supported. Introducing contrapositive reasoning is controversial in defeasible logic [9], but it is an intrinsic feature of classical logic. In other words, classical logic offers a nice solution to the problems raised in [8].

A more general approach to logic-based argumentation is to leave the logic for deduction as a parameter. This was proposed in abstract argumentation systems [25], and developed in assumption-based argumentation (ABA) [11]. A substantial part of the development of the theory and implement of $\mathrm{ABA}$ is focused on defeasible logic. However, ABA is a general framework allowing for the use classical logic for deduction, and thereby we could instantiate ABA with our proposal. 
It is also interesting to compare our approach with abstract argument systems. Superficially, an argument tree could be viewed as an argument framework in Dung's system. An argument in an argument tree could be viewed as an argument in a Dung argument framework, and each arc in an argument tree could be viewed as an attack relation. However, the way sets of arguments are compared is different.

Some differences between Dung's approach and our approach can be seen in the following examples.

Example 29. Consider a set of arguments $\left\{A_{1}, A_{2}, A_{3}, A_{4}\right\}$ with the attack relation $\mathscr{R}$ s.t. $A_{2} \mathscr{R} A_{1}, A_{3} \mathscr{R} A_{2}, A_{4} \mathscr{R} A_{3}$, and $A_{1} \mathscr{R} A_{4}$. Here there is an admissible set $\left\{A_{1}, A_{3}\right\}$. We can try to construct an argument tree with $A_{1}$ at the root. As a counterpart to the attack relation, we regard that $A_{1}$ is undercut by $A_{2}, A_{2}$ is undercut by $A_{3}$, and so on. However, the corresponding sequence of nodes $A_{1}, A_{2}, A_{3}, A_{4}, A_{1}$ is not an argument tree because $A_{1}$ occurs twice in the branch (violating Condition 2 of Definition 8). So, the form of the argument tree for $A_{1}$ fails to represent the fact that $A_{1}$ attacks $A_{4}$.

Example 30. Let $\Delta=\{b, b \rightarrow a, d \wedge \neg b, \neg d \wedge \neg b\}$, giving the following argument tree for $a$.

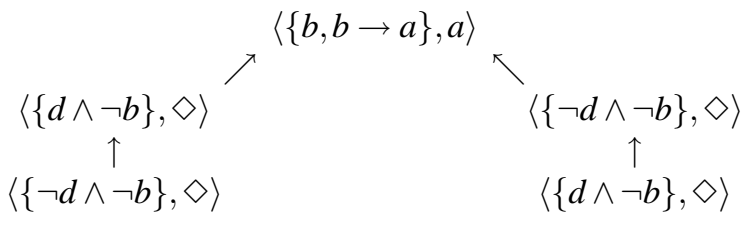

For this let $A_{1}$ be $\langle\{b, b \rightarrow a\}, a\rangle, A_{2}$ be $\langle\{d \wedge \neg b\}, \diamond\rangle$ and $A_{3}$ be $\langle\{\neg d \wedge \neg b\}, \diamond\rangle$. Disregarding the difference between the occurrences of $\diamond$, this argument tree rewrites as $A_{2} \mathscr{R} A_{1}, A_{3} \mathscr{R} A_{1}, A_{3} \mathscr{R} A_{2}$, and $A_{2} \mathscr{R} A_{3}$ where $A_{1}$ denotes the root node $\langle\{b, b \rightarrow a\}, a\rangle$. In this argument tree, each defeater of the root node is defeated. Yet no admissible set of arguments contains $A_{1}$.

Our proposal has been extended in a number of ways. As covered in [6], we have proposed techniques that take into account intrinsic aspects of the arguments and counterargument such as the degree of conflict between them, and the degree of similarity between them, and extrinsic aspects such as the impact on the audience and the empathy or antipathy the audience may have for individual arguments.

Further developments include formalization of enthymemes in our logic-based framework [16,7], a generalization of the framework to also consider the proponent of each argument, and thereby argue about whether a proponent is an appropriate proponent for that argument [17], and a refinement of the proposal to reason with temporal knowledge [18].

We have also generalised the proposal reviewed in this chapter to handle firstorder logic [4]. Indeed, this is straightforward since all we need to do is to use a first-order language and to use the classical first-order consequence relation instead of the classical propositional logic. The key finiteness results still hold: So for a finite number of formulae in the knowledgebase, there is a finite number of argument trees for a claim, and each of these trees contains a finite number of nodes. 
We have developed algorithms for generating arguments [12, 13], formalizations of decision problems concerning arguments and counterarguments in quantified Boolean formaulas [2], and compilation techniques with the aim of improving the viability of argumentation based on classical logic [5].

\section{Acknowledgements}

We wish to thank Maria Vanina Martinez for feedback on an earlier draft of this chapter.

\section{References}

1. L. Amgoud and C. Cayrol. On the acceptability of arguments in preference-based argumentation. In G. Cooper and S. Moral, editors, Proceedings of the 14th Conference on Uncertainty in Artificial Intelligence (UAI 1998), pages 1-7. Morgan Kaufmann, 1998.

2. Ph. Besnard, A. Hunter, and S. Woltran. Encoding deductive argumentation in quantified boolean formulae. Technical Report DBAI-TR-2008-60, Database and Artificial Intelligence Group, Institute of Information Systems, Technischen Universität Wien, 2008.

3. Ph. Besnard and A. Hunter. A logic-based theory of deductive arguments. Artificial Intelligence, 128:203-235, 2001.

4. Ph. Besnard and A. Hunter. Practical first-order argumentation. In Proceedings of the 20th National Conference on Artificial Intelligence (AAAI 2005), pages 590-595. MIT Press, 2005.

5. Ph. Besnard and A. Hunter. Knowledgebase compilation for efficient logical argumentation. In Proceedings of the 10th International Conference on Knowledge Representation (KR 2006), pages 123-133. AAAI Press, 2006.

6. Ph. Besnard and A. Hunter. Elements of Argumentation. MIT Press, 2008.

7. E. Black and A. Hunter. Using enthymemes in an inquiry dialogue system. In Proceedings of the Seventh International Joint Conference on Autonomous Agents and Multi-Agent Systems (AAMAS'08), pages 437-444. ACM Press, 2008.

8. M. Caminada and L. Amgoud. An axiomatic account of formal argumentation. In Proceedings of the 20th National Conference on Artificial Intelligence (AAAI 2005), pages 608-613, 2005.

9. M. Caminada. On the issue of contraposition of defeasible rules. In Computational Models of Argument: Proceedings of COMMA 2008, pages 109-115. IOS Press, 2008.

10. C. Chesñevar, A. Maguitman, and R. Loui. Logical models of argument. ACM Computing Surveys, 32:337-383, 2000.

11. P. Dung, R. Kowalski, and F. Toni. Dialectical proof procedures for assumption-based admissible argumentation. Artificial Intelligence, 170:114-159, 2006.

12. V. Efstathiou and A. Hunter. Algorithms for effective argumentation in classical propositional logic. In Proceedings of the International Symposium on Foundations of Information and Knowledge Systems (FOIKS'08), volume 4932 of LNCS, pages 272-290. Springer, 2008.

13. V. Efstathiou and A. Hunter. Focused search for arguments from propositional knowledge. In Computation Models of Argument: Proceedings of COMMA 2008, pages 159-170. IOS Press, 2008.

14. J. Fox, P. Krause, and M. Elvang-Gøransson. Argumentation as a general framework for uncertain reasoning. In Proceedings of the 9th Conference on Uncertainty in Artificial Intelligence (UAI 1993), pages 428-434. Morgan Kaufmann, 1993.

15. A. García and G. Simari. Defeasible logic programming: An argumentative approach. Theory and Practice of Logic Programming, 4:95-138, 2004. 
16. A. Hunter. Real arguments are approximate arguments. In Proceedings of the 22nd AAAI Conference on Artificial Intelligence (AAAI'07), pages 66-71. MIT Press, 2007.

17. A. Hunter. Reasoning about the appropriateness of proponents for arguments. In Proceedings of the 23rd AAAI Conference on Artificial Intelligence (AAAI'08). MIT Press, 2008.

18. N. Mann and A. Hunter. Argumentation using temporal knowledge. In Computational Models of Argument: Proceedings of COMMA'08, pages 204-215. IOS Press, 2008.

19. D. Nute. Defeasible logics. In Handbook of Logic in Artificial Intelligence and Logic Programming, Volume 3: Nonmonotonic Reasoning and Uncertainty Reasoning, pages 355-395. Oxford University Press, 1994.

20. J. Pollock. How to reason defeasibly. Artificial Intelligence, 57:1-42, 1992.

21. H. Prakken and G. Sartor. Argument-based extended logic programming with defeasible priorities. Journal of Applied Non-classical Logic, 7:25-75, 1997.

22. H. Prakken and G. Vreeswijk. Logical systems for defeasible argumentation. In D. Gabbay, editor, Handbook of Philosophical Logic, pages 219-318. Kluwer, 2002.

23. G. Simari and R. Loui. A mathematical treatment of defeasible reasoning and its implementation. Artificial Intelligence, 53:125-157, 1992.

24. B. Verheij. Automated argument assistance for lawyers. In Proceedings of the 7th International Conference on Artificial Intelligence and Law (ICAIL 1999), pages 43-52. ACM Press, 1999.

25. G. Vreeswijk. Abstract argumentation systems. Artificial Intelligence, 90:225-279, 1997. 